\title{
Magnetoresistance of One-Dimensional Subbands in Tunnel-Coupled Double Quantum Wires
}

\author{
J. S. Moon, M. A. Blount*, J. A. Simmons, J. R. Wendt, S. K. Lyo, and J. L. Reno
}

Sandia National Laboratories

Albuquerque; New Mexico 87185-1415

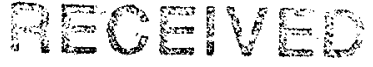

AU6 11899

$08 \mathrm{TI}$

We study the low-temperature in-plane magnetoresistance of tunnel-coupled quasione-dimensional quantum wires. The wires are defined by two pairs of mutually aligned split gates on opposite sides of a $\leq 1$ micron thick $\mathrm{AlGaAs} / \mathrm{GaAs}$ double quantum well heterostructure, allowing independent control of the width of each quantum well. In the ballistic regime, when both wires are defined and the field is perpendicular to the current, a large resistance peak at $\sim 6$ Tesla is observed with a strong gate voltage dependence. The data is consistent with a counting model whereby the number of subbands crossing the Fermi level changes with field due to the formation of an anticrossing in each pair of 1D subbands.

\footnotetext{
* Also at Dept. of Physics and Astronomy, Univ. of New Mexico.
} 


\section{DISCLAIMER}

This report was prepared as an account of work sponsored by an agency of the United States Government. Neither the United States Government nor any agency thereof, nor any of their employees, make any warranty, express or implied, or assumes any legal liability or responsibility for the accuracy, completeness, or usefuiness of any information, apparatus, product, or process disclosed, or represents that its use would not infringe privately owned rights. Reference herein to any specific commercial product, process, or service by trade name, trademark, manufacturer, or otherwise does not necessarily constitute or imply its endorsement, recommendation, or favoring by the United States Government or any agency thereof. The views and opinions of authors expressed herein do not necessarily state or reflect those of the United States Government or any agency thereof. 


\section{DISCLAIMER}

Portions of this document may be illegible in electronic image products. Images are produced from the best available original document. 
Coupled double two-dimensional electron systems (2DESs) have received much study in recent years, exhibiting a number of remarkable phenomena. In the weak tunneling regime, these include Coulomb drag [1] and an inter-layer tunneling Coulomb gap. [2] In the strong tunneling regime, an in-plane magnetic field-induced anticrossing of the two lowest energy 2D subbands has seen considerable study. [3-4] While most of this work has occurred in double quantum wells (DQWs), there has also been work in single QWs that are sufficiently wide $(\approx 1000 \AA)$ that a $2 \mathrm{DEG}$ forms at each interface of the QWs, so that it acts much like a DQW. [5]

Similar interesting new phenomena are expected to occur in coupled systems of lower dimensionality, such as coupled pairs of $1 \mathrm{D}$ wires or coupled OD quantum dots. Extensive work in coupled quantum dots has produced considerable evidence for coherent interdot coupling, whereby quantum dot "molecules" are formed. [6] By contrast, relatively little work has been done on coupled quantum wires (CQW). Early work examined CQWs defined in a single 2DES, whereby the coupling occurred laterally across a lithographically defined barrier. [7] More recently, vertically coupled quantum wires (VCQWs) were defined by mesa-etching a double quantum well heterostructure. [8] While this last approach enables a well-defined tunnel barrier, control over the 1D wire widths is limited, and in any event cannot be performed independently for the two wires.

In this paper, we present an experimental magnetotransport study of a pair of closely coupled VCQWs whose widths are independently tunable. Using our previously developed epoxy-bond-and-stop-etch (EBASE) technique [9] a pair of vertically aligned split gates is defined by electron beam lithography on each side of a double quantum well heterostructure whose thickness is only $0.8 \mu \mathrm{m}$. This use of close-proximity split gates on both sides of the epitaxial layers allows the width of each quantum wire to be 
independently controlled. By examining the conductance as a function of the various gate voltages, five different regimes are clearly identified. We define these regimes by the dimensionalities of the two channels provided by the top and bottom quantum wells: (I) 2D-2D; (ii) 2D-1D; (iii) 1D-1D; (iv) 1D—pinched-off; and (v) both pinched-off. In the presence of an in-plane magnetic field oriented perpendicular to the current direction at $\mathrm{T}=0.3 \mathrm{~K}$, we observe a large magnetoresistance peak at $\sim 6 \mathrm{~T}$ in the ballistic regime. The peak is strongly dependent on the gate voltages, and occurs only in regime (iii), where quasi-1D ballistic wires are defined in both quantum well channels. We attribute the magnetoresistance peak to the depopulation of 1D subbands in the VCQWs as magnetic-field-induced 1D-1D anticrossing develops. The data is in qualitative agreement with a recent theory by Lyo. [10]

The samples were fabricated from MBE-grown material consisting of two $200 \AA$ wide GaAs quantum wells separated by an $11 \AA \mathrm{Al}_{0.3} \mathrm{Ga}_{0.7}$ As barrier. For both the top and bottom quantum wells, a $1 \times 10^{12} \mathrm{~cm}^{-2} \mathrm{Si}$ delta doping layer was separated by a $700 \AA$ AlGaAs spacer layer. For both QWs, an additional $5 \times 10^{11} \mathrm{~cm}^{-2}$ Si delta-doping layer was placed $100 \AA$ away from the first delta layer. Diffused $A u / G e / N i$ made ohmic contact to both of the quantum wells. The EBASE process was used in conjunction with electron beam writing to fabricate aligned split gates of nominally identical dimensions on both sides of the epitaxial layers, as described in more detail elsewhere. [11] After the EBASE process, the top surface was $1450 \AA$ from the top QW, while the back surface was $6450 \AA$ from the bottom QW. Three devices were fabricated on a single sample. For all three, the split gates had openings of width $\mathrm{W}=4000 \AA$, while the lengths $\mathrm{L}$ were 1000,2000 , and $3000 \AA$. Figs 1 (a) and (b) show a top view and perspective view schematic of the sample structure, respectively, where the in-plane magnetic field (in the y-direction, for instance) is applied perpendicular to the measurement current. Fig. 1(c) shows a scanning electron micrograph of a cross section of a dual split-gate test structure, 
where sub-tenth-micron alignment accuracy between the front and back split gates is readily apparent.

Standard quantum Hall effect measurements on an identically EBASEd large area front- and back-gated Hall bar yielded electron densities in the two lowest $2 \mathrm{D}$ subbands of 2.5 and $1.15 \times 10^{11} \mathrm{~cm}^{-2}$, with no bias on the gates. A symmetric-antisymmetric energy gap of $\Delta_{\mathrm{SAS}}=1.7 \mathrm{meV}$ was measured, corresponding to the minimum density difference between the two lowest energy subbands obtained when the QWs are balanced, which occurred at a top gate bias of $0.45 \mathrm{~V}$. The total mobility of the structure is quite high at $5.8 \times 10^{5} \mathrm{~cm}^{2} / \mathrm{Vs}$, yielding an electron mean free path of $\sim 10 \mu \mathrm{m}$, indicating ballistic transport in the VCQWs. All measurements were performed using a standard lock-in technique with $5 \mathrm{nA}$ current at temperature $\mathrm{T}=0.3 \mathrm{~K}$ after brief illumination with an LED. Excellent reproducibility over thermal cycling was found.

In Fig. 2 we show the resistance of the $1000 \AA$ long device as a function of top split-gate voltage $V_{T}$, with back split-gate voltage $V_{B}=0$. A number of features appear which correspond to transitions between the different regimes described above. At $V_{T}=$ 0 , both channels are $2 \mathrm{D}$. As $\mathrm{V}_{\mathrm{T}}$ is made increasingly negative, the channel resistance shows a clear shoulder at $-0.35 \mathrm{~V}$ followed by a sharp increase at $-0.8 \mathrm{~V}$. These two features are due to the sequential depletion of first the top and then the bottom QWs in the regions directly beneath the top split gates. [13-14] Thus, at $\mathrm{V}_{\mathrm{T}} \approx-0.8 \mathrm{~V}$, where electrons directly beneath the top split gate become completely depleted from both QWs, coupled 1D channels are formed in both QWs. Hence this marks the transition from regime $\mathrm{ii}$, the $1 \mathrm{D}-2 \mathrm{D}$ case, to regime $\mathrm{iii}$, the $1 \mathrm{D}-1 \mathrm{D}$ case. We note that a simple capacitance calculation predicts the complete depletion of electrons beneath the split gates at $\mathrm{V}_{\mathrm{T}}=-0.9 \mathrm{~V}$, very close to the experimental value. 
As $V_{\mathrm{T}}$ is made further negative, we expect that both the widths and the electron densities of the quantum wires change [15], and that the quantum wire in the top QW narrows more rapidly than that in the bottom, since the channel depth is comparable to the gate opening. At $\mathrm{V}_{\mathrm{T}}=-2.35 \mathrm{~V}$, the channel resistance shows a weak plateau-like feature, where a definite change in the slope of the conductance curve is observed. [16] As $V_{T}$ is made yet more negative, a separate series of plateaux appear, corresponding to conductance steps quantized in units of $2 \mathrm{e}^{2} / \mathrm{h}$, until the channel is completely pinched off at $\mathrm{V}_{\mathrm{T}}=-3.9 \mathrm{~V}$. The monotonic quantization in steps of $2 \mathrm{e}^{2} / \mathrm{h}$ clearly indicates that only one channel is present for $-2.35 \mathrm{~V}>\mathrm{V}_{\mathrm{T}}>-3.9 \mathrm{~V}$. Thus the plateau at $-2.35 \mathrm{~V}$-which is clearly not part of the series of quantized steps—can be unambiguously identified as the point at which one of the two channels is pinched off. This point thus marks the transition from regime iii, the 1D-1D case, to regime iv, the 1D-pinched-off case. [17] We note that plots of the resistance vs. $V_{B}$ at fixed $V_{T}$ values appear very similar to Fig. 2 , except that the scale of the voltage axis is changed, so that the transition to regime iii, corresponding to formation of the VCQWs, occurs at $-4.4 \mathrm{~V}$, and both channels are pinched off at $-8.4 \mathrm{~V}$.

We now turn to the central result of this paper, the behavior of the VCQW devices in the presence of a magnetic field. As shown in Fig. 1(a), the field direction was perpendicular to both the growth direction and the VCQW current direction. Fig. 3(a) shows the magnetoresistance of the $1000 \AA$ long device when $V_{T}=V_{B}=0$ - i.e. in regime $\mathrm{i}$ where both channels are $2 \mathrm{D}$. The magnetoresistance exhibits a strong minimum at $\mathrm{B}=7 \mathrm{~T}$ followed by a weaker maximum at $8.5 \mathrm{~T}$. These are the well-known anticrossing features previously studied in large area double quantum well samples. [3] The in-plane B field displaces the two dispersion curves of the QWs by an amount $e B d / \hbar$, where $\mathrm{d}$ is the effective distance between the two electron layers. At sufficiently 
high B the curves anticross, opening a partial energy gap in the dispersion. The two features correspond to the upper and lower edges of the gap passing through the Fermi level, and occur due to the singularities in both the 2D density of states and the Fermi velocity at the upper and lower edges of the energy gap. We expect the two anticrossing features to be centered about a point $\mathrm{B}^{*}{ }_{2 \mathrm{D}}=\left(\sqrt{2 \pi n_{1}}+\sqrt{2 \pi n_{2}}\right) \hbar / e d$. Using the measured dark density values, we obtain $\mathrm{B}^{*} \approx 6.5 \mathrm{~T}$, in fair agreement with the data. The fact that the measured $\mathrm{B}^{*}{ }_{2 \mathrm{D}}$ is slightly higher is consistent with a slight increase in density due to the brief LED illumination.

As $V_{\mathrm{T}}$ is made more negative but remains below the transition to regime iii at -0.8 $\mathrm{V}$, the $2 \mathrm{D}$ anticrossing features persist. Fig. 3(b) shows the magnetoresistance for $\mathrm{V}_{\mathrm{T}}=-$ $0.7 \mathrm{~V}$ and $\mathrm{V}_{\mathrm{B}}=0$. The minimum remains at $7 \mathrm{~T}$, but the background resistance has increased. In addition, it appears that a very weak broad resistance peak is superimposed on the $2 \mathrm{D}$ anticrossing features. As will be made clear below, this marks the beginning of the formation of the VCQW.

Fig. 3(c) shows the magnetoresistance for $\mathrm{V}_{\mathrm{T}}=-0.8 \mathrm{~V}$ and $\mathrm{V}_{\mathrm{B}}=0$. This is just after the transition to regime iii, when the VCQW is formed and the system is 1D-1D. Although $\mathrm{V}_{\mathrm{T}}$ differs by only $0.1 \mathrm{~V}$ from Fig. 3 (b), the behavior of the magnetoresistance is dramatically different. A large broad peak in resistance appears, of magnitude $\sim 85 \Omega$. The peak is centered near $6.5 \mathrm{~T}$, slightly lower than the value observed in the 2D-2D case at $\mathrm{V}_{\mathrm{T}}=0$. The peak is also considerably wider than the $2 \mathrm{D}$ anticrossing features, with a full-width at half-maximum of $\sim 3.5 \mathrm{~T}$.

Fig. 3(d) shows the magnetoresistance at $V_{T}=-2.5 \mathrm{~V}$ and $V_{B}=0$, at which point the device has moved to regime iv and only a single $1 \mathrm{D}$ channel is occupied. At this point the resistance peak has completely disappeared. (As will be shown below, the peak 
remains present for values $-2.5 \mathrm{~V}<\mathrm{V}_{\mathrm{T}}<-0.8 \mathrm{~V}$ when $\mathrm{V}_{\mathrm{B}}=0$.) This clearly illustrates that the magnetoresistance peak is present only when the coupled $1 D-1 D$ wires are defined, i. e. only in regime iii. This feature, which is so qualitatively different from that observed in the 2D-2D case, can thus be considered to be an intrinsic property of VCQW transport.

In Fig. 4 (a)-(b), we plot the conductance $G=1 / R$ as a function of $B$ at several top gate voltages $V_{T}$, with a fixed $V_{B}=0$. As shown in Fig. 4(a), at $V_{T}=-1.0 \mathrm{~V}$, the zerofield conductance is $24\left(2 \mathrm{e}^{2} / \mathrm{h}\right)$. As $\mathrm{V}_{\mathrm{T}}$ becomes increasingly negative and the top QW wire narrows, the zero-field conductance monotonically decreases and reaches $8\left(2 \mathrm{e}^{2} / \mathrm{h}\right)$ at $\mathrm{V}_{\mathrm{T}}=-2.5 \mathrm{~V}$, when the top wire has completely pinched off. The dip in the magnetoconductance becomes smaller in amplitude as $\mathrm{V}_{\mathrm{T}}$ is made more negative, until disappearing completely at $V_{T}=-2.5 \mathrm{~V}$. As indicated by the dotted line, the position $B_{\min }$ of the broad minimum moves to increasingly lower values as $\mathrm{V}_{\mathrm{T}}$ becomes more negative. In Fig. 4(b), the in-plane magnetoresistance measurements at several backside gate voltages $V_{B}$ with fixed $V_{T}=-1.5 \mathrm{~V}$ show a similar behavior, but the shift in $B_{\min }$ as a function of $V_{B}$ occurs more strongly.

In Fig. 5 we show data from the 0.2 and $0.3 \mu \mathrm{m}$ long VCQW devices, here plotted as magnetoconductance rather than magnetoresistance. The data from these longer devices are very similar to that described thus far for the $0.1 \mu \mathrm{m}$ long device, except that the size of the features is reduced considerably. In the case of the $0.3 \mu \mathrm{m}$ device, the magnetoconductance peak observed at $\mathrm{V}_{\mathrm{T}}=-0.7 \mathrm{~V}$ evolves similarly into a broad dip at $\mathrm{V}_{\mathrm{T}}=-0.8 \mathrm{~V}$. Because the dip is shallower than in the $0.1 \mu \mathrm{m}$ device, a superimposed remnant of the $2 \mathrm{D}-2 \mathrm{D}$ conductance peak, presumably originating in the $2 \mathrm{D}$ leads, is relatively larger and hence gives the feature the appearance of a double minimum. 
We now turn to a discussion of the origin of the resistance peak. In a recent experiment by the Nottingham group [8], a similar broad resistance peak at an in-plane magnetic field $B=10 \mathrm{~T}$, perpendicular to the current, was observed in a VCQW defined by mesa etching. The wire had a lithographic width of $800 \mathrm{~nm}$ and length of $1.0 \mu \mathrm{m}$, and was fabricated in material with an elastic mean free path of $\sim 10 \mu \mathrm{m}$. In that work, the resistance peak was attributed to a semi-classical size effect, in which increased diffusive scattering occurs at the edges of the quantum wire. It was argued that for a field perpendicular to the current, the low-velocity electrons near the saddle-point of the fielddistorted dispersion curve play a larger role in the conductance, producing the enhanced resistance peak.

However, it is unclear whether our data can be explained by this proposed size effect. First, we note that the electron mean free path in the bulk, $\sim 10 \mu \mathrm{m}$, is much longer than the lithographic channel length of $0.1 \mu \mathrm{m}$. While some shortening of the mean free path is known to occur in quasi-1D structures, gate defined single quantum wires of 0.1 $\mu \mathrm{m}$ length in similarly high mobility material typically exhibit ballistic conductance. Indeed, that transport in our device is ballistic is further supported by our observation of quantized conductance steps when only one wire is occupied. Second, gate-defined electrostatic boundaries generally exhibit specular boundary scattering rather than diffusive. [18] If the observed magnetoresistance peak arose due to significant diffusive boundary scattering, then one would expect that the peak would be absent for ballistic wires, and would gradually become more pronounced as the length of the wire increased. [19] This is inconsistent with what we observe. 
Rather, we propose a model based on counting the number of $1 \mathrm{D}$ subbands crossing the Fermi level. [10] In a ballistic 1D wire, each occupied 1D subband contributes $2 \mathrm{e}^{2} / \mathrm{h}$ to the total conductance, and simply counting the number of occupied 1D subband yields the total conductance. Because this holds true irrespective of the form of the $1 \mathrm{D}$ dispersion, it also applies to ballistic VCQWs, but with more complicated energymomentum dispersion curves characterized by a $\Delta_{S A S}$ and $1 \mathrm{D}$ subband spacing $\Delta \mathrm{E}_{1 \mathrm{D}}$. Fig. 6 shows idealized sketches of the dispersion curves at different in-plane B fields in the case of $\Delta_{\mathrm{SAS}} \gg \Delta \mathrm{E}_{1 \mathrm{D}}$. In Fig. 6(a) the $\mathrm{B}=0 \mathrm{~T}$ case is shown, where the 1D dispersion curves for the symmetric subbands (solid lines) and antisymmetric subbands (dashed lines) are illustrated. When an in-plane magnetic field is applied perpendicular to the current direction, the 1D subband dispersion curves in one wire will shift in $\mathrm{k}$-space with respect to those in the other wire. Due to the tunnel coupling, at sufficiently high B the 1D dispersion curves from one wire will anticross with their counterparts from the other wire, opening a quasi gap of $\Delta_{\mathrm{SAS}}$, similar to the 2D case. [3] (The Fermi level will lie in the center of the Nth subband's anticrossing gap when $\mathrm{B}^{*}{ }_{1 \mathrm{D}}(\mathrm{N})=\left(k_{F 1}+k_{F 2}\right) \hbar / e d$, where $\mathrm{k}_{\mathrm{F} 1}$ and $\mathrm{k}_{\mathrm{F} 2}$ are the Fermi wave vectors of the Nth 1D subbands.) As depicted in Fig. 6(b), the resulting dispersion curve thus consists of several anticrossed pairs of $1 \mathrm{D}$ subbands characterized by a $\Delta_{S A S}$ and $1 \mathrm{D}$ subband spacing $\Delta \mathrm{E}_{\mathrm{ID}}$. Due to these multiple anticrossings, the number of $1 \mathrm{D}$ subbands crossing the Fermi level-i.e. the conductance-becomes substantially modified in two ways: (1) Whenever the Fermi level falls within a 1D anticrossing gap, the conductance is reduced by $2 \mathrm{e}^{2} / \mathrm{h}$ from the uncoupled double quantum wire case. (2) Because for each pair of anticrossed 1D subbands there is a large increase in the density of states at the edges of the anticrossing gap, each anticrossed pair can accommodate many more electrons. Since particle number 
is constant, the Fermi level thus drops substantially and intersects fewer 1D subbands, resulting in a drop in conductance.

These two different mechanisms can be illustrated by considering two different extreme cases characterized by $\Delta_{\mathrm{SAS}}$ and $\Delta \mathrm{E}_{1 \mathrm{D}}$. For the case of only one occupied pair of subbands and $\Delta \mathrm{E}_{1 \mathrm{D}} \gg \Delta_{\mathrm{SAS}}$, the magnetoconductance dip is mainly due to mechanism (1). Here the conductance is $4 \mathrm{e}^{2} / \mathrm{h}$ at $\mathrm{B}=0$ where the Fermi level crosses the subbands at four Fermi points. At intermediate B such that the Fermi level lies in the anticrossing gap and thus crosses the subbands at only two Fermi points, the conductance drops to $2 \mathrm{e}^{2} / \mathrm{h}$. Finally, at high B the anticrossing gap lies well above the Fermi level, which again crosses the subbands at four Fermi points, yielding a conductance of $4 \mathrm{e}^{2} / \mathrm{h}$. On the other hand, in the second case of many occupied subbands and $\Delta \mathrm{E}_{\mathrm{ID}} \ll<\Delta_{\mathrm{SAS}}$, as shown in Fig. 6 , a similar dip in conductance at intermediate B occurs, but this time due to mechanism (2) mentioned above. As B is increased and the anticrossings form, the increased density of states causes each subband to accommodate more electrons and the Fermi level drops. As a result the higher energy subbands become depopulated, the Fermi level crosses fewer Fermi points, and the conductance drops. As the magnetic field increases further and the anticrossing gaps move above the Fermi level, the number of Fermi points increases, causing the conductance to increase. Once all the anticrossing gaps have moved above the Fermi level, the density of states for each subband in the energy range below the Fermi level recovers its $\mathrm{B}=0$ value, as shown in Fig. 6 (c). The conductance then saturates. The primary difference between the two mechanisms is the sharpness of the conductance changes.

Qualitatively, the simple picture described above provides fair agreement with the data. Although additional studies of longer wires are needed, the observed reduction in 
the size of the magnetoresistance peak with increasing wire length is consistent with our model, since fewer electrons will traverse the wire ballistically. We note that the shift of $B_{\min }$ to lower field with increasingly negative $V_{T}$ in Fig. 4(a) is also consistent with the model. An increasingly negative $V_{T}$ not only narrows the width of the top quantum wire, but also raises the bottom of the wire's potential, reducing the electron density in all its 1D subbands. This results in a decrease in $\mathrm{k}_{\mathrm{F}}$, and hence a decreasing $\mathrm{B}^{*}{ }_{1 \mathrm{D}}$, for each pair of coupled 1D subbands, resulting in a decreasing $B_{\min }$. A similar decrease in $B_{\min }$ occurs with increasingly negative $V_{B}$, only significantly stronger, as shown in Fig. 4(b). This stronger decrease of $B_{\min }$ with negative $V_{B}$ is consistent with the back split gates being about four times further away from the VCQW. In that case the electric field lines from the gates are oriented more vertically at the VCQW, causing a more rapid rise in the bottom of the VCQW's potential as its width decreases with negative gate bias. [15]

Finally, although no direct quantitative comparison is available at present, preliminary calculations [10] show behavior very similar to our data. This further supports our conclusion that the observed magnetoresistance peak occurring at fields of a few $T$ is an intrinsic Fermi-surface related property of a ballistic coupled 1D-1D system. We note that recent theoretical work predicts a possible edge-related effect in VCQWs due to widely differing widths of the top and bottom quantum wires [20], and also a large in-plane magnetoconductance enhancement in VCQWs in the strongly diffusive regime [21]. These topics clearly constitute intriguing areas for future investigations in coupled 1D quantum wires.

The authors gratefully acknowledge N. E. Harff for discussions and W. E. Baca for technical assistance. This work was supported by the U.S. DOE under Contract DEAC04-94AL85000. Sandia is a multi-program laboratory operated by Sandia Corporation, a Lockheed-Martin company. 


\section{References}

[1] T.J. Gramila, J. P. Eisenstein, A. H. MacDonald, L. N. Pfeiffer, and K. W. West, Phys. Rev. Lett. 66, 1216 (1991)

[2] J. P. Eisenstein, L. N. Pfeiffer, and K. W. West, Phys. Rev. Lett. 69, 3804 (1992).

[3] J. A. Simmons, S. K. Lyo, N. E. Harff, and J. F. Klem, Phys. Rev. Lett. 73, 2256 (1994); A. Kurobe, I.M. Castleton, E.H. Linfield, M.P. Grimshaw, K.M. Brown, D.A. Ritchie, M. Pepper, and G.A.C. Jones, Phys. Rev. B 50, 4889 (1994).

[4] G. S. Boebinger, A. Passner, L. N. Pfeiffer, and K. W. West, Phys. Rev. B 43, 12673 (1991).

[5] T. Ihn, H. Carmona, P. C. Main, L. Eaves, and M. Henini, Phys. Rev. B 54, R2315 (1996); H. C. Manoharan, Y. W. Suen, T. S. Lay, M. B. Santos, and M. Shayegan, Phys. Rev. Lett. 79, 2722 (1997).

[6] D. G. Austing, T. Honda, K. Muraki, Y. Tokura, and S. Tarucha, Physica B 251, 206 (1998); T. Schmidt, R. J. Haug, K. V. Klitzing, A. Forster, and H. Luth, Phys. Rev. Lett. 78, 1544 (1997); F. R. Waugh, M. J. Berry, D. J. Mar, R. M. Westervelt, K. L. Campman, and A. C. Gossard, Phys. Rev. Lett. 75, 705 (1995).

[7] N. Tsukada, A. D. Wieck, and K. Ploog, Appl. Phys. Lett. 56, 2527 (1990), C. C. Eugster, J .A. Delalamo, M. J. Rooks, and M. R. Melloch, Appl. Phys. Lett. 64, 3157 (1994).

[8] M. J. Gompertz, T. Ihn, P. C. Main, A. Nogaret, L. Eaves, M. Henini, and S. P. Beaumont, Physica B 249-251, 162 (1998).

[9] M. V. Weckwerth, J. A. Simmons, N. E. Harff, M. E. Sherwin, M. A. Blount, W. E. Baca, and H. C. Chui, Superlattices and Microstructures 20, 561 (1996).

[10] S. K. Lyo, private communication. 
[11] J. R. Wendt, J. A. Simmons, J. S. Moon, W. E. Baca, M. A. Blount, and J. L. Reno, Semicond. Sci. Technol. 13, A86 (1998).

[12] The measured $\Delta_{\mathrm{SAS}}$ is in good agreement with the value of $1.4 \mathrm{meV}$ obtained by a Hartree simulation of the growth structure.

[13] X. Ying, S. R. Parihar, H. C. Manoharan, and M. Shayegan, Phys. Rev. B 52, R11611 (1995).

[14] J. S. Moon et al., Appl. Phys. Lett. 74, 314 (1999); S. Luryi, ibid. 52, 501 (1988).

[15] S. E. Laux, D. J. Frank, and F. Stern, Surf. Sci. 196, 101 (1988); Z. Wu and P. P. Ruden, J. Appl. Phys. 74, 6234 (1993); Y. Sun and G. Kirczenow, J. Appl. Phys. 77, $6361(1995)$

[16] Beside the clear indication of the slope change in conductance, the systematic movement of the feature was found from $-1.7 \mathrm{~V}$ in dark to $-2.3 \mathrm{~V}$ with LED illumination.

[17] Plots similar to Fig. 2 taken at increasingly negative values of $V_{B}$ show a proportional shift of the entire resistance vs. $V_{T}$ curve towards less negative $V_{T}$ values, supporting the general picture of the behavior of the VCQWs described in the text.

[18] H. van Houton et al., Surf. Sci. 196, 144 (1988); J. S. Moon, J. A. Simmons, and J. L. Reno, Appl. Phys. Lett. 71, 656 (1997).

[19] In the limit of the purely diffusive boundary scattering with a mean free path $1>\mathrm{W}$, the resistivity is proportional to $(1 / \mathrm{W}) / \mathrm{n}(1 / \mathrm{W})$. See also T. J. Thornton, M. L. Roukes, A. Scherer, and B. P. Van de Gaag, Phys. Rev. Lett. 63, 2128 (1989).

[20] O. E. Raichev, and F. T. Vasko, Phys. Rev. B 57, 12350 (1998).

[21] S. K. Lyo, J. Phys.:Condens Matter 8, L703 (1996). 


\section{Figure Captions}

Fig. 1. (a) Top view, and (b) perspective view schematic diagrams of the sample, consisting of a Hall bar geometry mesa with mutually aligned submicron split gates on both the front and back sides. (b) Scanning electron micrograph of a cross-section of a dual split-gate test structure of similar dimensions to the actual sample. After the EBASE process, the topside is against the epoxy and buried beneath the epitaxial layers.

Fig. 2 Resistance vs. top gate bias $\mathrm{V}_{\mathrm{T}}$, at back bate bias $\mathrm{V}_{\mathrm{B}}=0$, for the $\mathrm{L}=0.1 \mu \mathrm{m}$ and $\mathrm{W}=0.4 \mu \mathrm{m}$ sample. The left and right plots have different scales for the resistance and cover different ranges of $\mathrm{V}_{\mathrm{T}}$. Inset is an expanded view of the $-0.5 \mathrm{~V}<\mathrm{V}_{\mathrm{T}}<0$ range. Arrows indicate the transitions between different regimes: $2 \mathrm{D}-2 \mathrm{D}$ to $1 \mathrm{D}-2 \mathrm{D}$ at $-0.35 \mathrm{~V} ; 1 \mathrm{D}-2 \mathrm{D}$ to $1 \mathrm{D}-1 \mathrm{D}$ at $-0.8 \mathrm{~V}$; and 1D-1D to $1 \mathrm{D}$-pinched off at $-2.35 \mathrm{~V}$.

Fig. 3. Resistance of $0.1 \mu \mathrm{m}$ long device vs. magnetic field perpendicular to both current and growth direction, for fixed back gate voltage $V_{B}=0$ and different top gate voltages, (a) $0 \mathrm{~V}$, (b) $-0.7 \mathrm{~V}$, (c) $-0.8 \mathrm{~V}$, and (d) $-2.5 \mathrm{~V}$. For all cases the resistance scale spans roughly $20 \%$ of the $B=0$ resistance.

Fig.4. Conductance vs. in-plane magnetic field of $0.1 \mu \mathrm{m}$ long device, for (a) several $\mathrm{V}_{\mathrm{T}}$ voltages at a fixed $V_{B}=0$, and (b) several $V_{B}$ voltages at a fixed $V_{T}=-1.5 \mathrm{~V}$. Dashed lines are guides to the eye indicating the position in $\mathrm{B}$ of the conductance minimum.

Fig. 5. Conductance vs. in-plane magnetic field of (a) $0.2 \mu \mathrm{m}$ and (b) $0.3 \mu \mathrm{m}$ long 
devices at several top gate voltages, for a fixed $V_{B}=0$. The $1 D-1 D$ conductance dips are considerably weaker than for the $0.1 \mu \mathrm{m}$ long device. For both of the devices shown here, when $\mathrm{V}_{\mathrm{T}}<-0.7 \mathrm{~V}$ the dip in conductance appears to have a double minimum due to a weak 2D-2D anticrossing conductance peak from the leads being superimposed.

Fig. 6 The dispersion curves for the coupled ballistic 1D-1D wires are sketched for $\Delta E_{1 D}$ $\ll \Delta_{\mathrm{SAS}}$ when the in-plane B field is applied in the y-direction with (a) $\mathrm{B}=0 \mathrm{~T}$, (b) $\mathrm{B}$ $=\mathrm{B}^{*}{ }_{1 \mathrm{D}}$ for the lowest subbands and (c) $\mathrm{B} \gg \mathrm{B}^{*}{ }_{\mathrm{DD}}$. The symmetric and antisymmetric subbands are shown as solid lines and dashed lines, respectively. The lowest subband branches are indicated by thicker lines. The Fermi level, indicated by a dashed horizontal line, drops significantly from (a) to (b) due to the change of density of states. (The position of the Fermi level is not exact.) The conductance is given simply by the occupied Fermi points, indicated by the black dots. 


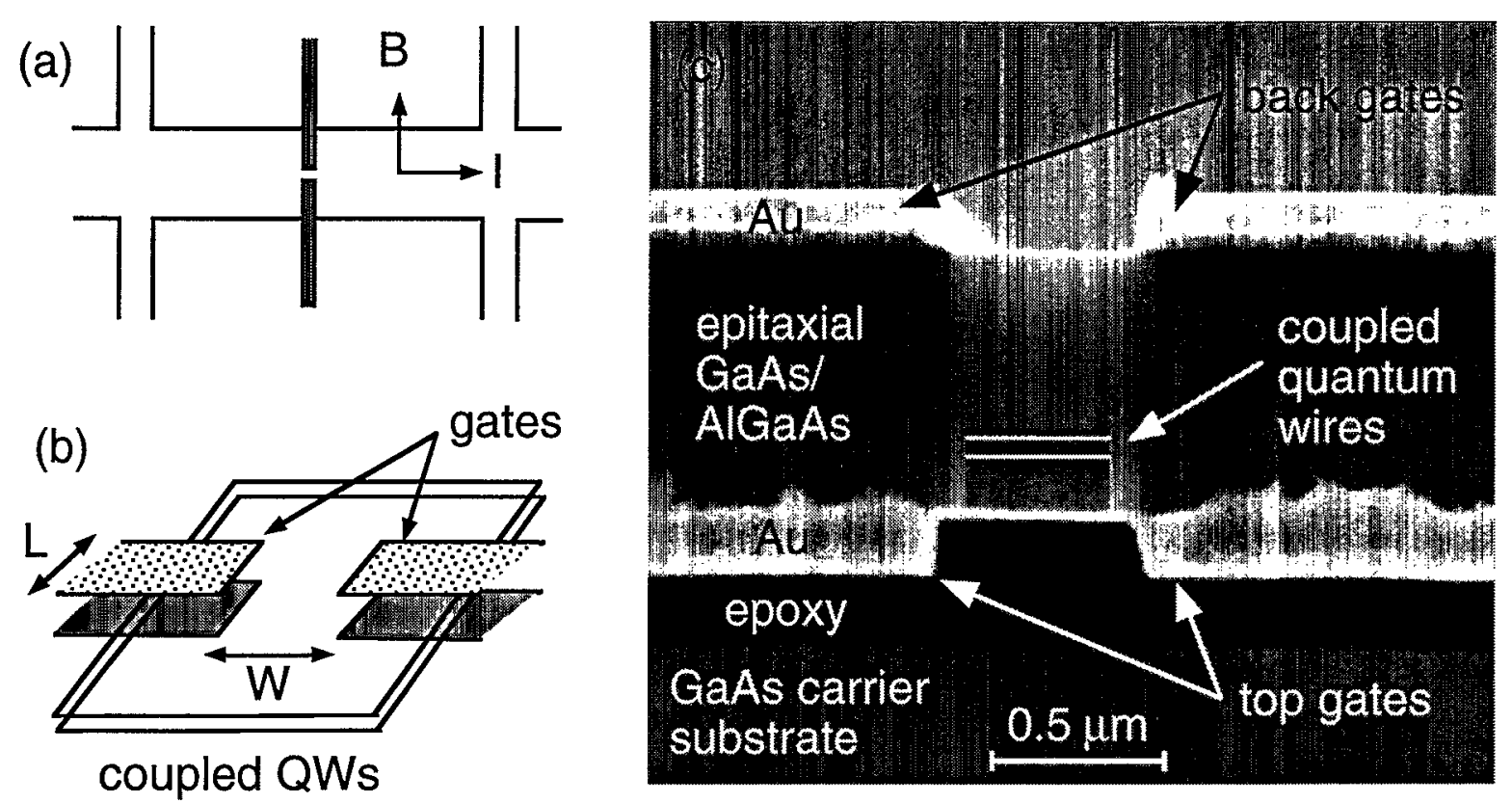

Fig. 1

Moon et al., PRB 


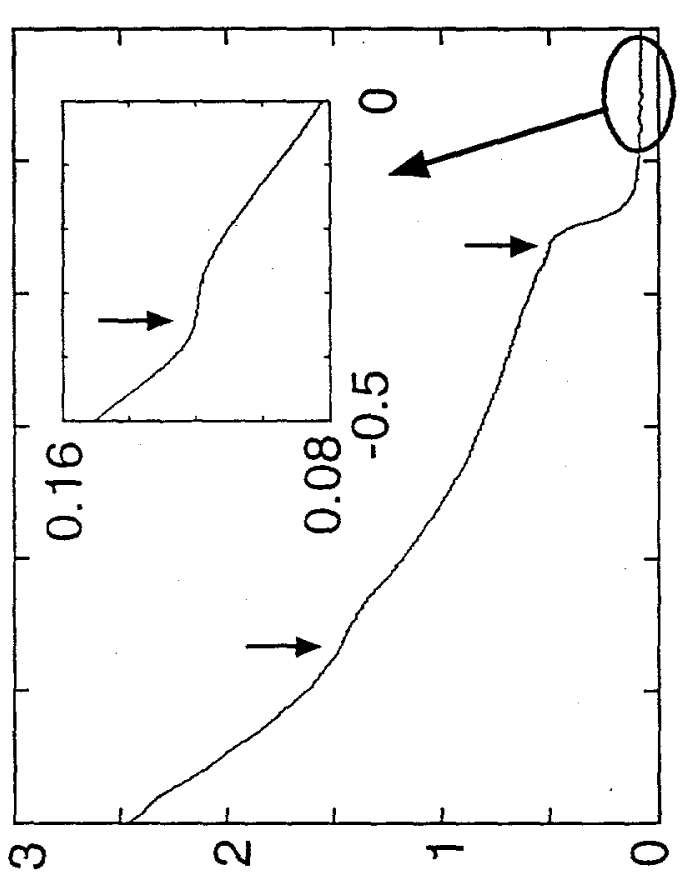

0

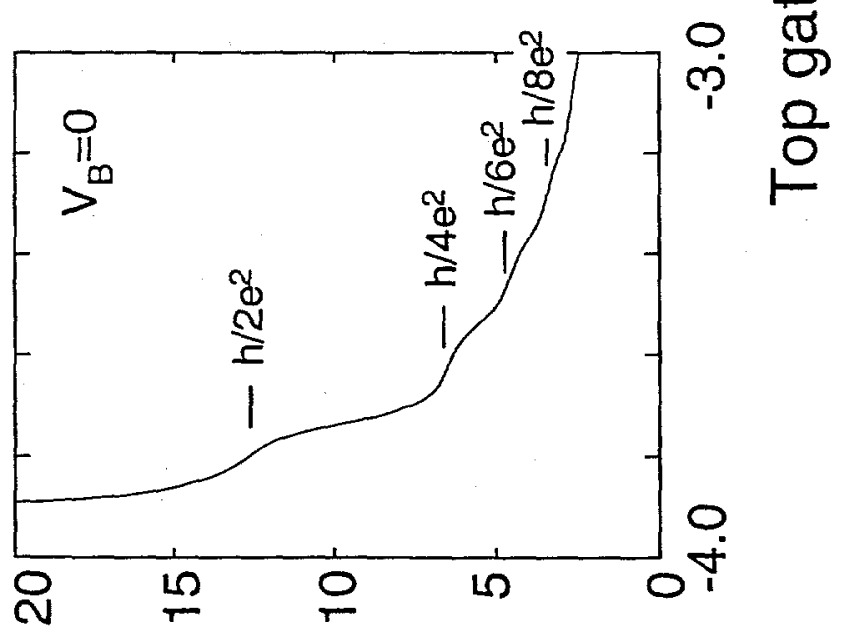

(Ју) әouels!səy 


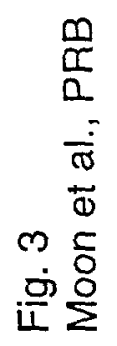
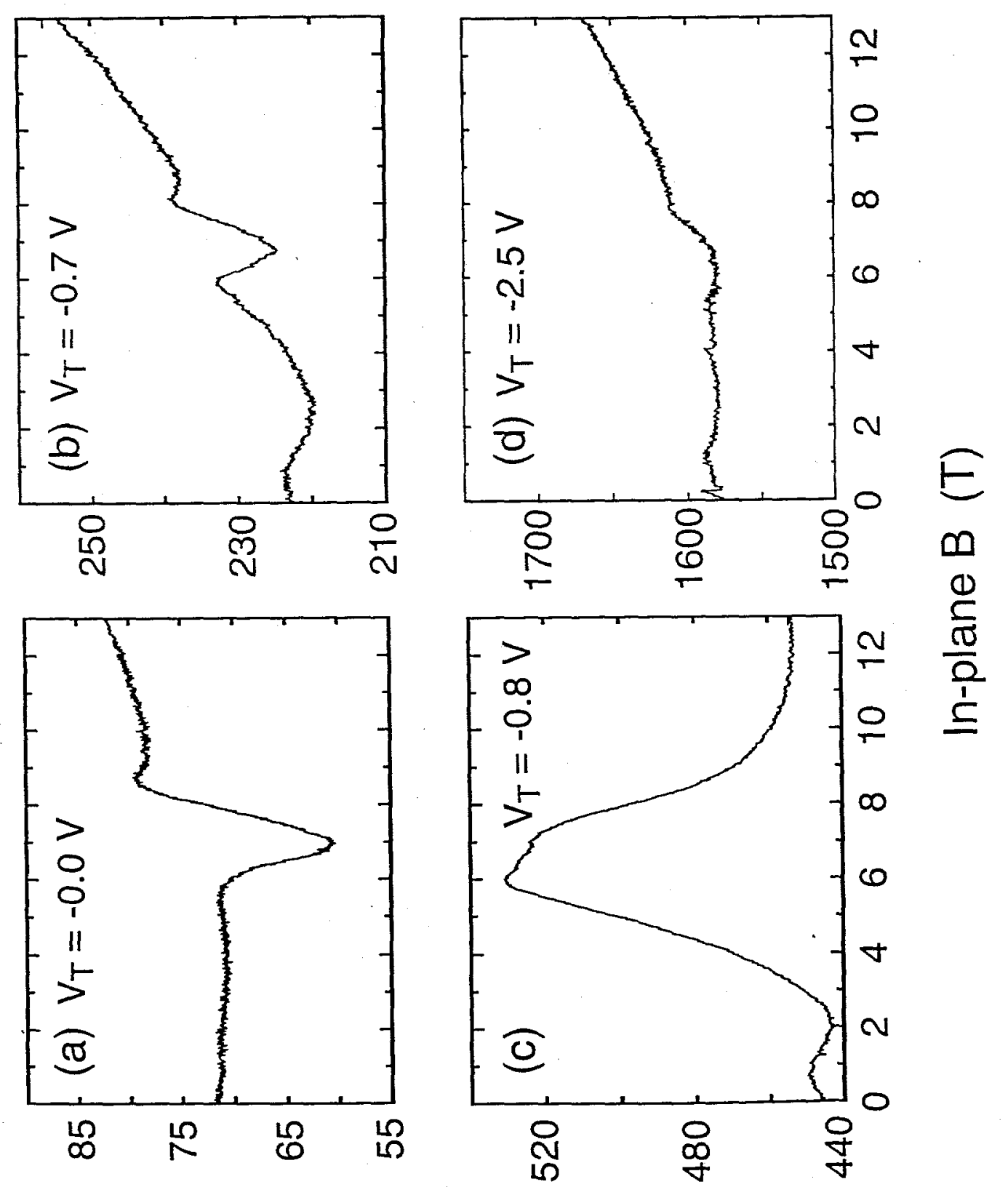

(๘) әouełs!səy 

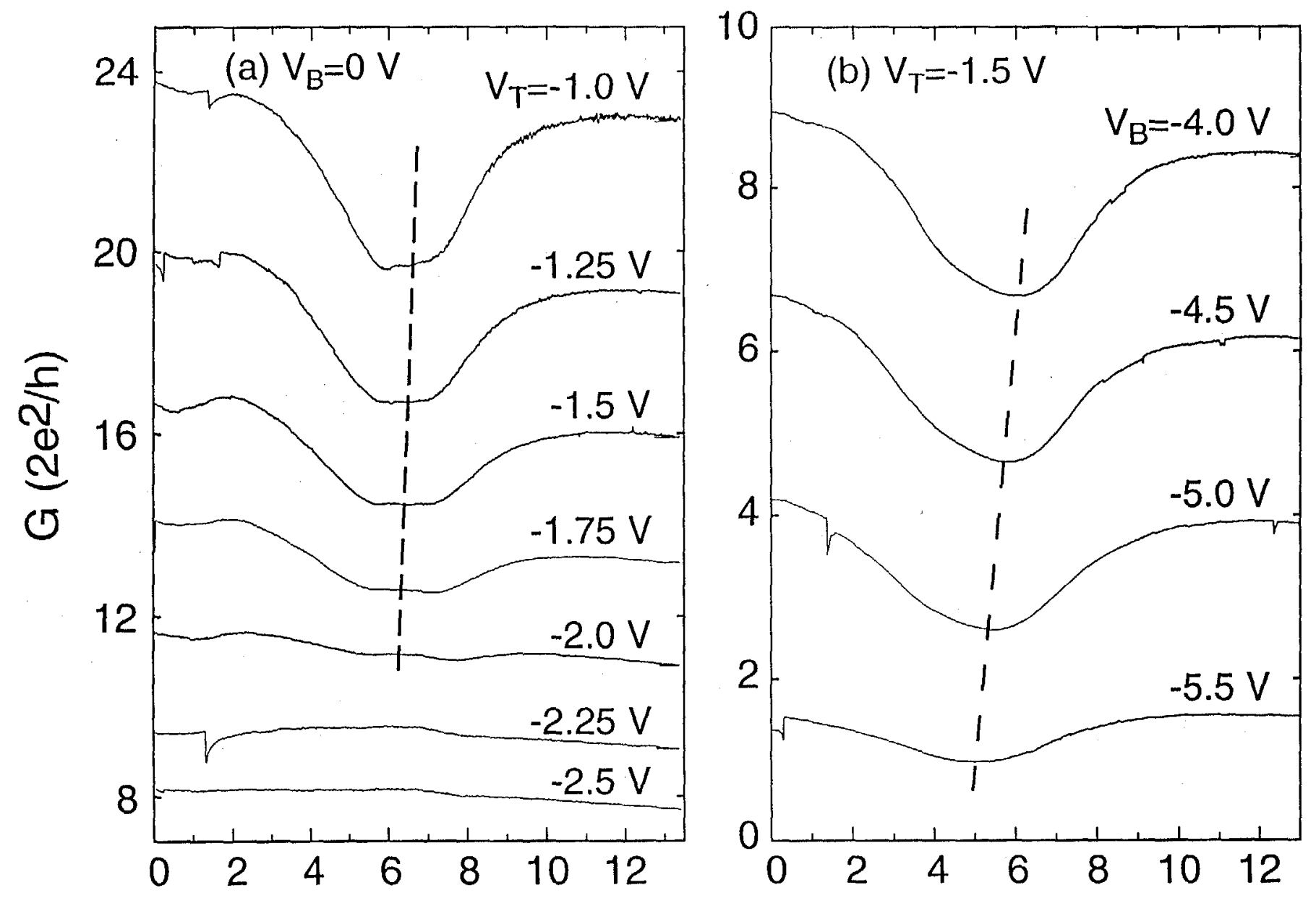

In-plane B (T) 


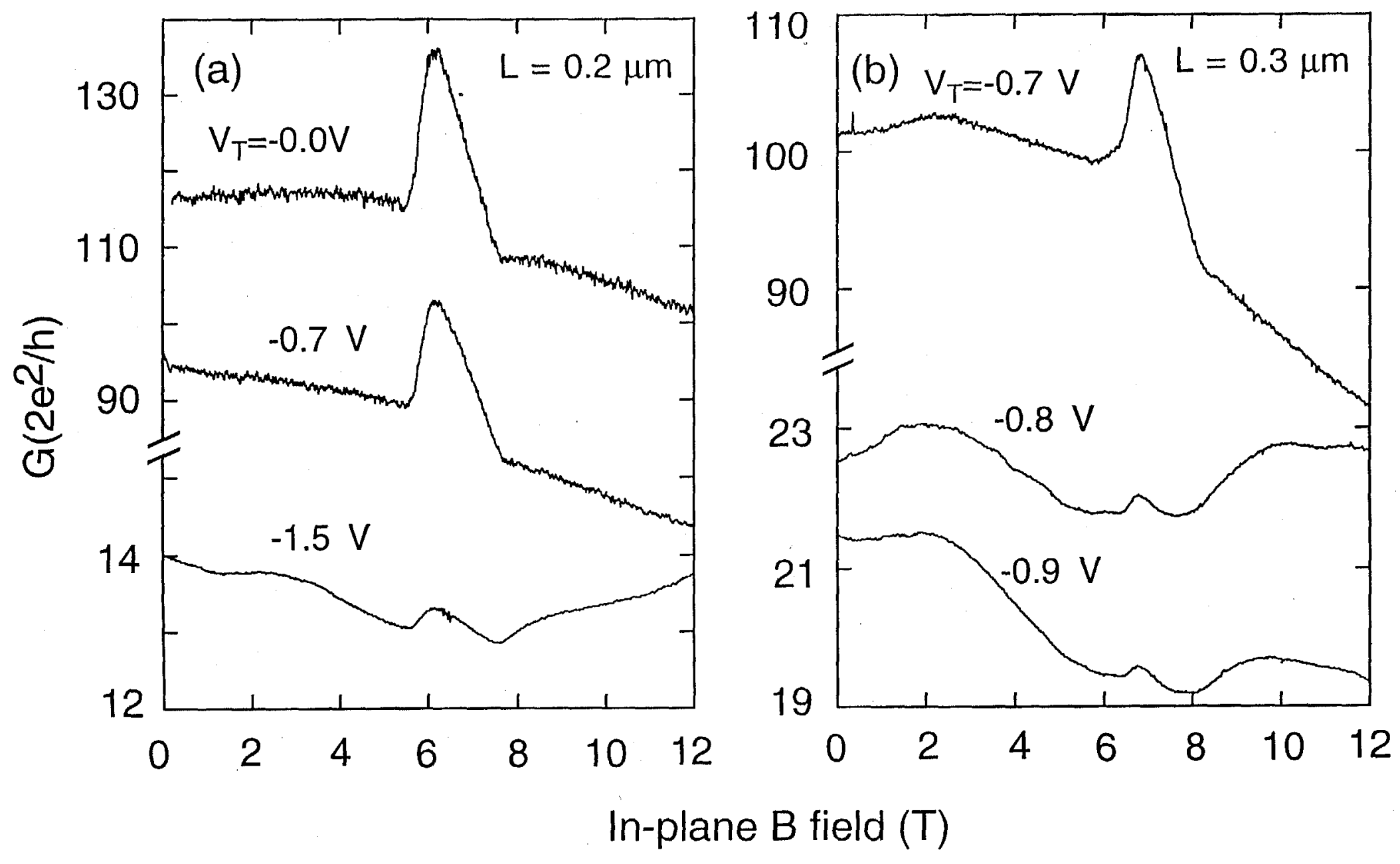

Fig. 5

Moon et al., PRB 


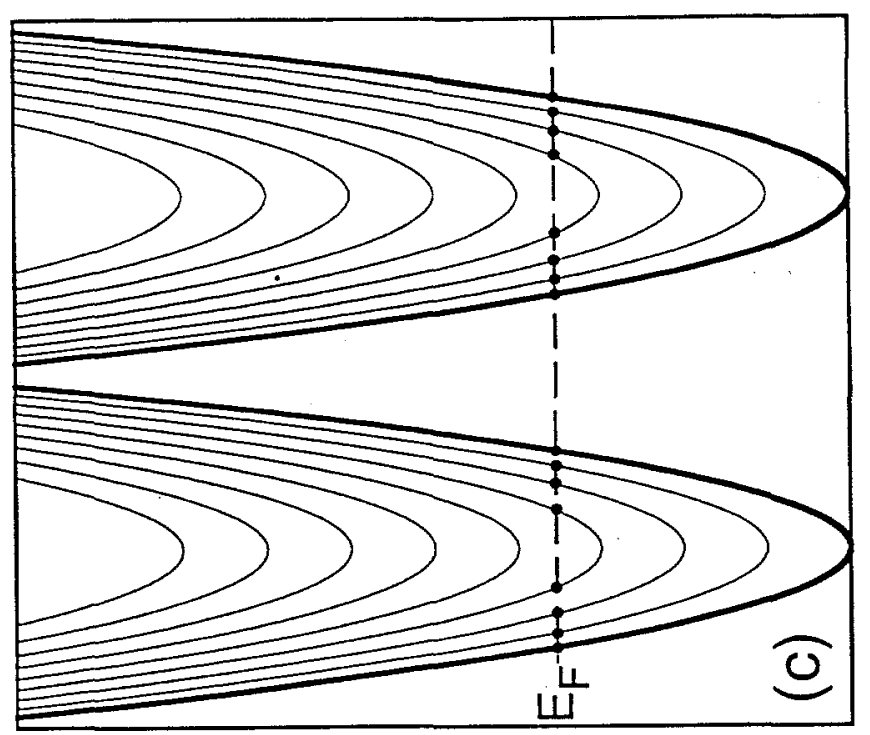

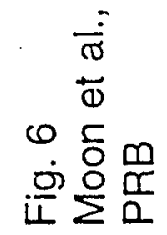
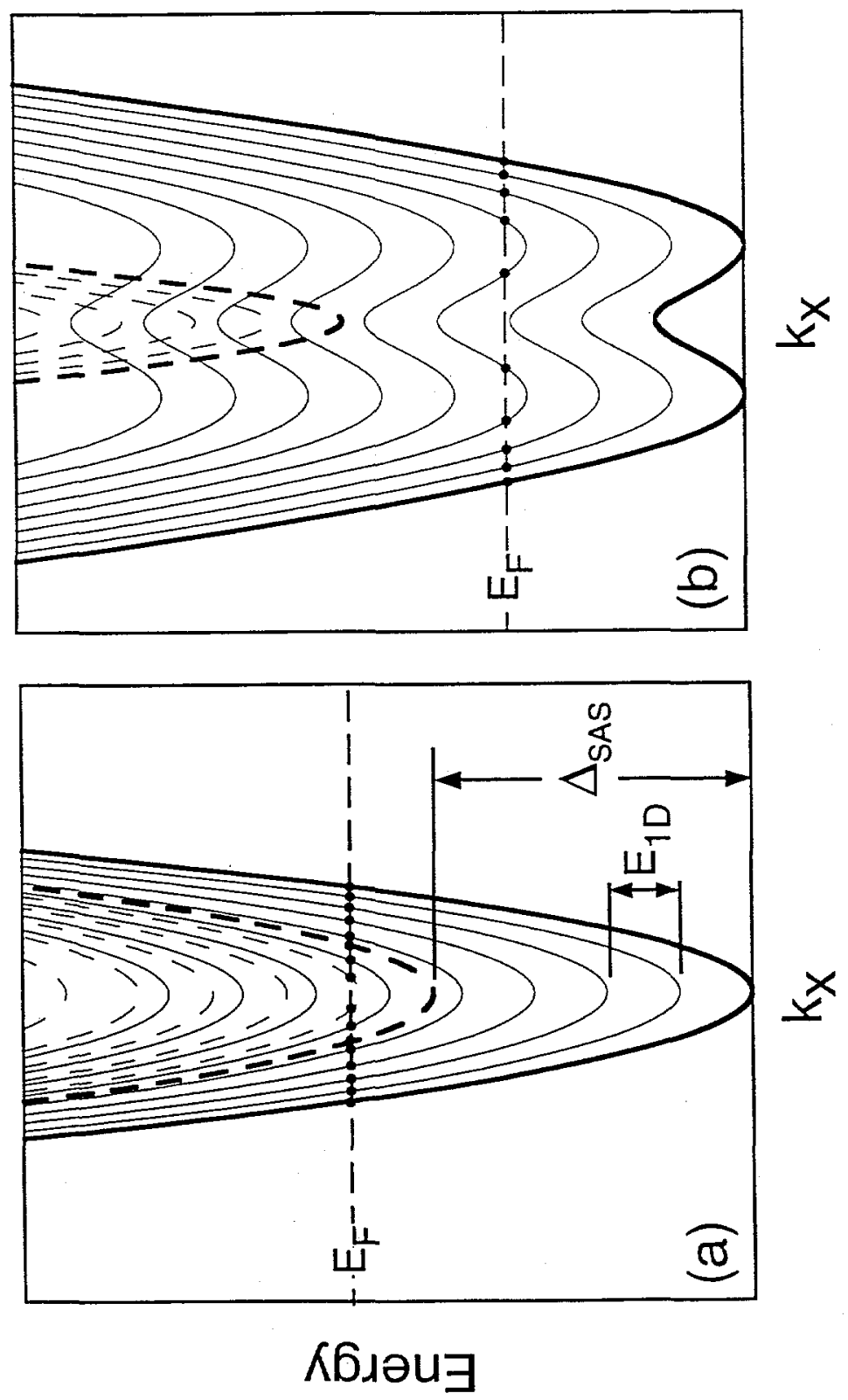